\title{
Exposure to traffic air pollutants in a major US urban area with high freeway traffic: has anything changed over the past decade?
}

\author{
S. A. Grinshpun, M. Yermakov, L. A. Grinshpun, T. Reponen, \\ M. Simmons, P. H. Ryan \& G. K. LeMasters \\ Center for Health-Related Aerosol Studies, \\ University of Cincinnati, USA
}

\begin{abstract}
While airborne particulate matter (PM) has been associated with numerous health effects, very few studies have extensively examined the changes in exposure to PM and its health-relevant constituents, which occur in major metropolitan areas over time (e.g., 5-10 years). This study addresses changes in the PM2.5 concentration and elemental composition between two monitoring campaigns (2002-2005 and 2010-2011) carried out in the Cincinnati (Ohio, USA) metropolitan area. The area is known for high traffic volume, largely represented by diesel-powered trucks on regional freeways. The air monitoring was conducted at four sites. Through an analysis of variance comparison, differences between the two data sets were assessed for PM2.5, ten selected elements, elemental carbon (EC), organic carbon (OC), and EC/OC (a surrogate of the diesel exhaust contribution). Measured at the site that was previously found to exhibit the highest level of EC attributable to traffic, most of traffic related elements and the EC/OC ratio showed statistically significant decreases in concentration over time; however, the decrease became of borderline significance when wind variables were incorporated into the model. No major differences between data generated in the two campaigns were observed at the other monitoring stations with respect to EC/OC. Although the positive trends identified in this study (e.g., decrease in PM2.5, Ti, V, Mn, Fe, $\mathrm{Zn}, \mathrm{Br}$, and $\mathrm{Pb}$, $\mathrm{EC}, \mathrm{OC}$, and $\mathrm{EC} / \mathrm{OC}$ ) may become sustainable in the future, no strong evidence was found that the air quality control and engine exhaust control policies implemented between 2005 and 2010 have produced significant changes in traffic air pollution levels in the entire metropolitan area. The PM monitoring
\end{abstract}


results are being compared to the public perception data acquired through a specially-developed survey administered to 100 residents of the region. The data will allow establishing an association between the public perception about the ambient air quality evolution and the actual measured changes in traffic-related pollution levels.

Keywords: traffic air pollution, aerosol exposure monitoring, PM2.5, carbon, elemental concentration.

\section{Introduction}

In urban areas, vehicular traffic usually represents a major source of ambient air pollution. Exposure to aerosol particles emitted by car and truck engines has been associated with adverse heath effects among children and adults. Among these health effects is the exacerbation of existing asthma in populations residing near freeways [1]. Epidemiological studies aiming at establishing exposurehealth relationships often rely on air monitoring data collected over a limited time period while the health data collection usually takes much longer time. Thus, it is important to determine whether the initially collected exposure data are still relevant beyond the air monitoring period and can be adequately utilized in the development of exposure-health relationships. This may or may not be the case considering various environmentally friendly policies which have recently been (and continue being) implemented to control emission from heavy-duty vehicles and reduce the number of traffic congestion zones. These interventions aim at decreasing the concentration of ambient traffic aerosols. At the same time, other interventions, such as widening freeways by adding lanes often significantly increase the traffic volume. Very few studies have extensively examined the changes in exposure to health-relevant traffic-originated particulate matter (PM) occurring in major metropolitan areas during a substantial time period (e.g., 5-10 years).

In this investigation, we examined changes in the PM2.5 concentration and elemental composition between two monitoring campaigns carried out in 20022005 and 2010-2011 within the Cincinnati (Ohio, USA) metropolitan area, which is recognized for intensive diesel-truck traffic and high prevalence of asthma and respiratory allergies. Additionally, we developed a survey to assess the public perception regarding the ambient air quality evolution in the tested area and to compare the findings with the air monitoring data.

\section{Method}

The tested area (the city of Cincinnati and its suburbs) has an annual diesel truck traffic volume of $\sim 10$ million on regional freeways and is commonly referred to as "allergy valley". With a total population of over 2 million people, it represents a mid- to large-size North American metropolitan area. Four air sampling sites, featuring different levels of traffic air pollution $[2,3]$ and spread over the entire area, were selected for the study. Among these are: an urban downtown site (close to a high-traffic freeway; previously identified to have high levels of 
elemental carbon attributable to traffic, ECAT [3]), a riverbank site (close to a medium-traffic freeway, mid-level of ECAT) and two suburban sites that have different proximity to major freeways and low-to-medium level of ECAT.

The sites were used in the 2002-2005 and 2010-2011 ambient air monitoring campaigns which generated a total of 507 daily PM2.5 samples subjected to different analyses, including gravimetric, elemental, and carbon (organic, OC, and elemental, EC). These 24-hour samples were collected on weekdays with precipitation below $1 / 2$ inch starting approximately at 9 AM. The sampling was not conducted on days when a major road construction or other non-ordinary particle emitting activities were taking place.

At each site, a Harvard-type PM2.5 impactor (MS\&T Area Sampler, Air Diagnostics and Engineering, Inc., Harrison, ME, USA) mounted on a tripod under rain-protective covers operated at a flow rate of $20 \mathrm{~L} / \mathrm{min}$. The particles were collected on a 37-mm quartz filter (Whatman Inc., Clifton, NJ, USA) which was analyzed for EC and OC using the Thermal-Optical Transmittance. The EC/OC-ratio (a surrogate of the aerosol diesel emission) was determined for each site on each monitoring day. Based on these data, the geometric mean (GM) and the geometric standard deviation (GSD) were calculated at each site for each of the two monitoring campaigns. The differences in EC/OC between the two campaigns at the four sites were determined using analysis of variance (ANOVA). These were regarded as statistically significant if p-value was below 0.0125 , which is a traditional p-value of 0.05 adjusted using the Bonferroni adjustment made for the 4-site comparison: $\mathrm{p}_{\mathrm{adj}}=0.05 / 4=0.0125$. An additional Harvard-type PM2.5 impactor was deployed at the downtown location; this once was equipped with a $37-\mathrm{mm}$ Teflon membrane filter (pore size $=1 \mu \mathrm{m}$ ) that was subjected to a gravimetric analysis (generating the daily PM2.5 mass concentration data) and an X-Ray Fluorescence analysis (generating the elemental mass concentration data for a total of 38 elements, including those representing the traffic emission and the regional ambient aerosol background).

Wind speed and direction were recorded for each day; the wind index was calculated for each sample. The wind index is a rescaling of the angle between two vectors: from the nearest major traffic source to the site and predominant wind direction to a scale of zero to one. This index is a continuous variable; sites directly upwind of the nearest traffic source have a wind index of zero; sites directly downwind of the nearest traffic source have a wind index of 1 ; and sites perpendicular to the wind direction have an index of 0.5. Analyses were performed using two approaches: with and without wind data included in the model.

\section{Results, discussion and future effort}

The EC concentrations decreased from 2002-2005 through 2010-2011 at all four sites. However, this decrease was not statistically significant, regardless whether the wind variables were included. The EC/OC-ratios (presented in Table 1) show a declining trend at three of the four sites. Among those three, only at one site (downtown, featuring the highest level of ECAT) was the decrease statistically 
significant and only if the wind data were not included into the model. The $\mathrm{EC} / \mathrm{OC}$ decrease became of borderline significance when the wind variables were incorporated. The overall PM2.5, as well as most of traffic related elements (EC, OC, Ti, V, Mn, Fe, Zn, Br, and Pb) sampled at the downtown site, showed the same trend.

Although the positive trends identified in this study may become sustainable in the future, we found no strong evidence that the air quality control policies and engine exhaust control measures implemented between 2005 and 2010 have produced significant changes in traffic air pollution levels within the entire metropolitan area (possibly, with one exception - the downtown site)..

In parallel to the air monitoring data comparison, we developed a survey aiming at assessing the public perception about the ambient air quality evolution between 2002-2005 and 2010-2011. This survey is being administered among 100 residents of the Cincinnati metropolitan area, who are being asked to predict how the concentration of traffic (diesel) air pollutants in their area changed for this 5-10-year period. The comparison of the information acquired through this survey to the air monitoring data obtained in the two campaigns will allow establishing an association between the public perception about the ambient air quality evolution and the actual measured changes in traffic-related pollution levels.

Table 1: Comparison of EC/OC values from the databases collected in 2002-2005 and 2010-2011 at four monitoring sites.

\begin{tabular}{|c|c|c|c|c|c|c|}
\hline \multirow{2}{*}{ Monitoring site } & \multicolumn{2}{|c|}{ EC/OC: GM (GSD) } & \multicolumn{3}{c|}{ ANOVA comparison } \\
\cline { 2 - 7 } & $\begin{array}{c}\text { Campaign } \\
\text { 2002-2005 }\end{array}$ & $\begin{array}{c}\text { Campaign } \\
2010-2011\end{array}$ & \multicolumn{2}{|c|}{$\begin{array}{c}\text { Without the wind } \\
\text { index }\end{array}$} & \multicolumn{2}{|c|}{ With the wind index } \\
\cline { 4 - 8 } & $p_{\text {adj }}$ & $\begin{array}{c}\text { Significant } \\
\text { difference? }\end{array}$ & $p_{\text {adj }}$ & $\begin{array}{c}\text { Significant } \\
\text { difference? }\end{array}$ \\
\hline $\begin{array}{c}\text { Downtown } \\
\text { (close to high- } \\
\text { traffic freeways) }\end{array}$ & $\begin{array}{c}0.256 \\
(1.34)\end{array}$ & $\begin{array}{c}0.179 \\
(1.61)\end{array}$ & 0.0021 & yes & 0.0164 & $\begin{array}{c}\text { No or } \\
\text { borderline }\end{array}$ \\
\hline $\begin{array}{c}\text { Riverbank (close } \\
\text { to a medium- } \\
\text { traffic freeway) }\end{array}$ & $\begin{array}{c}0.114 \\
(1.45)\end{array}$ & $\begin{array}{c}0.131 \\
(1.47)\end{array}$ & 0.515 & no & 0.379 & no \\
\hline $\begin{array}{c}\text { Suburb (close } \\
\text { but upwind from } \\
\text { a medium-traffic } \\
\text { freeway) }\end{array}$ & $\begin{array}{c}0.145 \\
(1.35)\end{array}$ & $\begin{array}{c}0.113 \\
(1.57)\end{array}$ & 0.0561 & no & 0.212 & no \\
\hline $\begin{array}{c}\text { Suburb (far from } \\
\text { all freeways, } a \\
\text { background site) }\end{array}$ & $\begin{array}{c}0.106 \\
(1.57)\end{array}$ & $\begin{array}{c}0.102 \\
(1.61)\end{array}$ & 0.814 & no & 0.728 & no \\
\hline
\end{tabular}




\section{Conclusions}

Declining trends were identified between the ambient air monitoring campaigns taking place in 2002-2005 and 2010-2011 in Cincinnati for most of trafficrelated elements as well as the EC/OC-ratio (a surrogate of the aerosol diesel emission). The data analysis revealed, however, that the achieved air quality improvement fell short of statistical significance for the entire tested metropolitan area. The borderline significance found for some comparisons suggests that the positive trends identified in this study may become sustainable in the future. Thus, the air monitoring should continue in the tested area to quantitatively characterize the benefits of the traffic emission control policies which are currently in place. The preliminary results from the public perception survey showed that the residents' opinions may not always adequately reflect the changes in traffic-related pollution levels observed from the air monitoring.

\section{Acknowledgements}

The authors are grateful for the support from the National Institute for Environmental Health Sciences (USA) through grants RO1 ES11170 and R21 ES017957 as well as the pilot grants from the University of Cincinnati's Research Council and the Center for Sustainable Urban Environments.

\section{References}

[1] Holguin, F. Traffic, outdoor air pollution, and asthma. Review, Immunology and Allergy Clinics of North America, 28, pp. 577-588, 2008.

[2] Martuzevisius, D., Grinshpun, S.A., Reponen, T., Górny, R.L., Shukla, R., Lockey, J.E., Hu, S., McDonald, R., Biswas, P., Kliucininkas, L., and LeMasters, G., Spatial and temporal variation of PM2.5 concentration throughout an urban area with high freeway density - the Greater Cincinnati study, Atmospheric Environment, 38, pp. 1091-1105, 2004.

[3] Ryan, P., LeMasters, G., Biswas, P., Levin, L., Hu, S., Lindsey, M., Bernstein, D., Lockey, J., Villareal, M., Hershey, G.K.K, and Grinshpun, S.A., A comparison of proximity and land use regression traffic exposure models and wheezing in infants, Environmental Health Perspectives, 115(2), pp. 278-284, 2007. 